Relations industrielles

Industrial Relations

\title{
Un Collège ouvrier
}

\section{Fernand Jolicoeur}

Volume 7, numéro 4, septembre 1952

URI : https://id.erudit.org/iderudit/1023033ar

DOI : https://doi.org/10.7202/1023033ar

Aller au sommaire du numéro

\section{Éditeur(s)}

Département des relations industrielles de l’Université Laval

\section{ISSN}

0034-379X (imprimé)

1703-8138 (numérique)

Découvrir la revue

Citer ce document

Jolicoeur, F. (1952). Un Collège ouvrier. Relations industrielles / Industrial Relations, 7(4), 310-311. https://doi.org/10.7202/1023033ar

Tous droits réservés @ Département des relations industrielles de l’Université Laval, 1952
Ce document est protégé par la loi sur le droit d'auteur. L'utilisation des services d'Érudit (y compris la reproduction) est assujettie à sa politique d'utilisation que vous pouvez consulter en ligne.

https://apropos.erudit.org/fr/usagers/politique-dutilisation/ 


\section{INFORMATIONS}

\section{UN COLLEGE OUVRIER}

La C.T.C.C. inaugurera à Québec, en novembre prochain, un Collège ouvrier dont les cours dureront quatre semaines. C'est la première fois au pays qu'on tentera une expérience d'éducation ouvrière aussi longue. Jusqu'à présent, les différent's mouvements syndicaux ont tenu des sessions intensives répétées de trois ou quatre jours, parfois même de dix jours. Mais il était impossible, dans une aussi courte période, de donner une formation à la fois assez large et assez précise pour rencontrer les besoins multiples des militants ouvriers. Pourra-t-on le faire dans un mois? Nous savons bien que cela serait encore illusoire. Mais nous voulons faire ce pas de plus vers l'établissement d'une école permanente de syndicalisme oi: nous pourrions préparer un plus grand nombre de chefs ouvriers.

\section{Evolution du MOUVEMENT OUVRIER CANADIEN}

Le mouvement ouvrier canadien est maintenant parvenu à l'àge adulte. Les deux guerres mondiales et la crise économique, comme la situation bouleversée qui a suivi ces évènements et qui semble vouloir se prolonger encore longtemps, a mis le syndicalisme à l'épreuve et en a fait un élément de base dans l'évolution radicale de la vie sociale et économique qui se produit sous nos yeux. Par son ascension, il a été amené à préciser ses buts et à prendre des positions bien définies.

Il a exigé une participation de plus en plus active de la classe ouvrière à la vie de l'entreprise, de la profession et de la nation. Il a indiqué clairement que cette participation doit s'élargir jusqu'à une collaboration franche et libre avec les autres groupes sociaux. Il n'a jamais senti plus qu'aujourd'hui le besoin urgent de trouver dans les rangs des ouvriers eux-mêmes, les chefs capables de le conduire vers sa véritable destinée qui nest pas seulement la revendication de conditions de travail plus humaines, mais la réforme des structures mêmes de la société.

\section{N'́́.CESSITÉ D'UN COLLÈGE}

A mesure que les ouvriers prennent conscience des nouvelles responsabilités de leur classe dans un monde de plus en plus industrialisé, ils exigent avec raison que leur mouvement leur fournisse l'occasión d'acquérir une formation économique et sociale leur permettant de faire face à leurs problèmes et de remplir les fonctions multiples qui peuvent leur être confiées. Nous ne pouvons plus nous contenter de dispenser uniquement les éléments nécessaires à une action syndicale immédiate. Nous devons ouvrir des horizons aussi vastes que le monde et donner le goût d'une culture aux proportions de l'humanité. Un Collège ouvrier qui mérite ce nom doit maintenant être une grande école qui met autant de soin à bâtir ses programmes d'études, qu'à choisir ses professeurs et à trier sur le volet ses élèves.

\section{Cours au programme}

Les principaux cours au programme de ce Collège ouvrier de la C.T.C.C. porteront sur l'histoire sociale, économique et syndicale, sur l'économie politique telle qu'elle s'exprime dans la réalité canadienne, sur la législation ouvrière fédérale et provinciale, sur les techniques syndicales, sur la doctrine sociale de l'Eglise et la déclaration de principes de la C.T.C.C.

Mais un bon nombre d'heures seront aussi consacrées à des cours plus pratiques, comme les méthodes d'éducation ouvrière, la propagande et la publicité, la procé- 
dure d'assemblée et l'art de la parole. Nous voulons, non seulement former des hommes capables de trouver des solutions à leurs problèmes économiques ou sociaux, mais aussi des moniteurs qui entraîneront leurs confrères de travail. La meilleure éducation ouvrière qui puisse se donner est celle qui se fait par les ouvriers eux-mêmes.

\section{MÉTHOde D'ENSEIGNEMENT}

La méthodè dont on se servira tiendra compte à la fois des sujets à l'étude, des ouvriers eux-mèmes et du but qu'on se propose d'atteindre.

Les sujets à l'étude sont des sujets pratiques, des problèmes humains qui demandent beaucoup plus de réflexion personnelle et commune qu'ils exigent d'exposés techniques et théoriques. Les ouvriers sont des adultes déjà aux prises avec les questions qu'on veut les aider à résoudre. Et l'on se propose comme but de faire prendre conscience tout de suite à la classe ouvrière du rôle qu'elle doit jouer dans la société et la rendre apte à jouer ce rôle immédiatement.

Notre méthode a donc besoin d'être active. Elle doit permettre à chacun dexprimer ses propres difficultés ou sa propre façon de voir. Elle doit habituer l'ouvrier à discuter et à chercher et trouver lui-même des solutions. Chaque exposé sera donc suivi d'une discussion. Certains cours comprendront des travaux pratiques exécutés par les élèves, par exemple argumentation pour demande d'augmentation de salaires devant un tribunal d'arbitrage; rédaction d'un petit journal pour les élèves; composition de circulaires et de pancartes, etc.

Les cours seront données par des professeurs de l'Université Laval, des spécialistes des questions au programme, des officiers et des permaments de la C.T.C.C. Les professeurs resteront à la disposition des élèves entre les heures de cours qui se donmeront dans l'édifice des syndicats catholiques de Québec où la C.T.C.C. a son siège social. Le programme couvrira les quatre semaines consécutives du mois de novembre, du lundi matin au vendredi soir. Pendant les fins de semaine, les élèves retourneront chez eux, du samedi matin au lundi matin. Un programme récréatif est prévu pour employer les temps libres, après les cours et las soirées du mardi et du jeudi qui seront toujours libres.

Ce Collège ouvrier s'adresse surtout à ceux qui ont déjà acquis un minimum de connaissances syndicales, par l'étude ou la pratique, soit en suivant des cercles d'études, des sessions d'Ecole d'Action ouvrière régionale, etc., soit en remplissant une charge d'officier, d'agent d'affaires, d'organisateur, de délégué de département, de responsable de service. Cette préparation élémentaire est nécessaire pour suivre avec avantage les cours plus poussés qui seront donnés.

\section{TÂCHE ONÉREUSE ET NÉCESSAIRE}

Cela coûtera cher de compenser le salaire d'une trentaine d'ouvriers qui laisseront l'usine pour tout un mois et de payer leur chambre, pension et frais de cours pour le même temps. Mais tous les organismes affiliés à la C.T.C.C. sont prêts à faire les sacrifices qu'une charge aussi lourde va leur imposer pour assurer au syndicalisme un avenir qui vaille les efforts qu'ils font aujourd'hui. Comme mouvement d'inspiration catholique, nous nous devons plus que tout autre de former des chefs à toute épreuve qui pourront diriger la lutte de la classe ouvrière pour sa libération en même temps que pour la paix véritable de toute la société. 